\title{
About the Influences of Some Major Biotic Drivers on the Population Dynamics in Host-parasite Systems: Interpretative Lessons from an Extended Nicholson-Bailey Model
}

\author{
Jean Béguinot ${ }^{1,2^{\star}}$ \\ ${ }^{1}$ Société Histoire Naturelle-Bourgogne Nature, 7 bvd H. P. Schneider, 71200, Le Creusot, France. \\ ${ }^{2}$ Université Bourgogne, 6, Boulevard Gabriel, 21000 Dijon, France.
}

Author's contribution

The sole author designed, analysed, interpreted and prepared the manuscript.

Article Information

DOI: $10.9734 / A R R B / 2020 / v 35 i 830256$

Editor(s):

(1) Dr. Paola Angelini, University of Perugia, Italy.

Reviewers:

(1) Mahabaleshwar G. Hegde, University of Agricultural Sciences, Dharwad (UASD), India. (2) Devinder Sharma, Sher-e-Kashmir University of Agricultural Sciences and Technology (SKUAST), India. Complete Peer review History: http://www.sdiarticle4.com/review-history/59302

\section{ABSTRACT}

Population dynamics within host-parasite systems in insects is governed by a series of factors, both endogenous and exogenous. Among them, five factors may be considered as major drivers: the respective inherent rates of increase of the host and of the parasite, the level of resource available to the host, the respective immigration rates of the host and of the parasite. While only the first two (the inherent rates of increase of host and parasite) are considered in the original Nicholson and Bailey model, an extended version of the model includes also the other three parameters, thus providing a broader (although still schematic) approach to the host-parasite population dynamics. A brief analysis of the respective influences of each of these five driving parameters on the main features of host-parasite dynamics is derived accordingly, based upon this extended model. Finally, specific attention is paid to the major concerns due to the cyclic outbreaks of both the host and the parasite, regarding in particular the amplitude, the periodicity and the conditions of onset of the cyclicity. Both the practical aspects of the cyclic regime and its possible adaptative significance are discussed. As a whole, this approach aims to provide some general clues for the interpretation of various features of the host-parasite dynamics, as reported from field observations. 
Keywords: Population dynamics; abundance fluctuation; cyclic outbreak; insect; pine processionary moth; Thaumetopoea pityocampa.

\section{INTRODUCTION}

Predator-prey relationships - or parasite and host relationships in the case of insects - are among the most ubiquitous forms of interspecific interactions within animal communities. The diversity and the frequent complexity of the reciprocal interactions between predator and prey species have therefore prompted strong speculative interest since a long time $[1,2]$. In some cases, the severe economic consequences resulting from insufficient control of preys by predators have also induced much practical concern as well $[3,4]$.

One major point, regarding the outcome of these interactions is the crudely contrasted patterns of variations of both predator and prey abundances across successive generations [5-7]. These contrasted, alternative patterns being either:

(i) A stable dynamic equilibrium between the respective abundances of the predator and the prey, with the equilibrium level depending, of course, on the relative performances (reproduction rate, detection / escape abilities ...) of each of the two partners involved, or,

(ii) A more or less strong, oscillating pattern between the respective abundances of the predator and the prey, with usually regular cycles showing periodic oscillations.

These two contrasted patterns are all the more remarkable since one or the other can be alternatively encountered within one and the same predator-prey (or parasite-host) system, involving the same couple of interacting species (Figs 1 to 3 ).

A series of more or less refined mathematical models have been developed, designed to account for the intermingled reciprocal interactions between predator and prey species. Models which, thereby, try to explain the causes involved in the alternative occurrence of one or the other of these two alternative patterns.

One of the more popular models, was originally proposed by Nicholson and Bailey [8] (reviewed in [5,9]). This model is focused on the entomological context, where, most often, the predator is a parasite (sensu lato, including parasitoid) and the prey is the correspondingly parasitized host. This model addresses the interactions between host and parasite in such a general and encompassing form that it still retains its current relevance and is still often taken as a reference. Yet, some important shortcomings in the original design of the model proposed by Nicholson and Bailey deserve specific consideration:

(i) As it is, the Nicholson and Bailey model addresses only the influences of the inherent rates of increase of the host and the parasite, while neglecting the role of the possible limitations in feeding resource available for the host, that may also greatly affect the corresponding host-parasite population dynamics; in other words, the original model addresses the bi-trophic level (i.e. host-parasite) rather than the tri-trophic level (i.e. plant-host-parasite) among mutual biotic relationships;

(ii) It does not take into account the metapopulation context, namely, the contribution to the local population dynamics of the immigration / emigration fluxes of the host or the parasite or both, coming from more or less distant other populations.

An extended form of the original Nicholson \& Bailey model is thus desirable, intended to jointly address (i) the possible limitation in the available resource for the host and (ii) the contributions of host and parasite dispersals among more or less distant populations. That is, in short, an extension of the original model which integrates both (i) the tri-trophic interactions (including both bottom-up and top-down regulations) and (ii) the surrounding metapopulation context (accounting thus for inter-populations dispersals).

This extension of the Nicholson \& Bailey model will be considered, and implemented hereafter, to serve the main purpose of the present study: trying to disentangle, thus highlight - and finally explain - the respective influences of the following factors on the pattern of variations of the host and the parasite abundances along successive generations:

(i) the influence of the inherent rate of increase of the host species,

(ii) the influence of the inherent rate of increase of the parasite species, 
(iii) the influence of the level of available resource for the host,

(iv) the influence of the net immigration rate of the parasite,

(v) the influence of the net immigration rate of the host.

With regard to the last two points (i.e. the contribution of the metapopulation context to host-parasite interactions), specific attention will be addressed to the role of the reciprocal exchanges of migrating individuals between more or less distant host and parasite populations on the amplitude and the period length of cyclic outbursts.

At last, theoretical predictions in this respect will be compared to field observations reported for defoliating insects [10].

\section{EXTENSION OF THE NICHOLSON \& BAILEY MODEL}

As mentioned above, the extended version of the Nicholson \& Bailey model accounts also for (i) bottom-up regulation due to possible limitation in resource for the host and (ii) the contribution of the metapopulation context via inter-populations emigration / immigration exchanges. The methodologic way according to which this enlarged version was derived is further described in more details in the Appendix; only the main factors involved in the model being specified below, for a better understanding of the following.

In term of host-parasite relationships, the relevant time unit to be considered is the generation duration (usually common to both the parasite and the host: [5]). Dedicated models are thus expressed in discrete-time, with the time increment ' $i$ ' labelling the successive generations of both the host and the parasite.

Let then ' $h$ ' and ' $p_{i}$ ' be the densities (numbers of individuals per unit area of field investigation) of the host and the parasite respectively, at the common generation labelled ' $i$ '. Consider now the respective own dynamics of the parasite species and of the host species - with the "own" dynamics of the host being understood as it would be in the absence of the parasite. These own dynamics are characterized quantitatively by the parameters below:

- For the parasite (predator) species, three parameters: ' $v$ ', 'f' and ' $a$ '. With ' $v$ ' as the average number of viable parasites issued from each parasitized host-individual, ' $f$ ' as the proportion of mated, egg-laying females within the population of parasites and 'a' as the mean foraging area for oviposition by a parasite female. In the following, this last parameter, ' $a$ ', has been chosen as the distinctive parameter, singularizing and thus differentiating the reproductive own performance of a given parasite species (of course the other influent factors, ' $f$ ' and / or ' $v$ ', could have been considered as well);

- For the host (prey) species, two parameters: ' $r$ ' and ' $K$ '. Parameter ' $r$ ' is for the inherent rate of increase of the host individual (that is, as it would be in the absence of any limitation in resource availability for the development of a host individual). Now, as in fact the available feeding resource for the host is always more or less limited in nature, it is necessary to consider also a parameter accounting for this limitation: ' $K$ ', the so-called "resource carrying capacity" for the host population. More precisely, ' $\mathrm{K}$ ' accounts, here, for the density of host individuals that could ultimately be fed by the hypothetically exhaustive consummation of the available resource.

In addition, let ' $e_{H}$ ' and ' $e_{P}$ ' be the additional contributions of immigration - more exactly the balance between immigration and emigration of the host individuals and of the parasite individuals respectively. ' $e_{H}$ ' and ' $e_{P}$ ' complement the host and the parasite densities, generated in situ, within the studied population system itself.

\section{STABLE AND OSCILLATORY DYNAMIC EQUILIBRIUM: TWO CONTRASTED PATTERNS, CHARACT- ERISTIC OF HOST-PARASITE DYNAMICS}

It has long been recorded that in predator-prey dynamics in general, and in host-parasite dynamics in particular, the relative abundances (densities) of each the two interacting species progressively reach dynamic equilibrium values respectively. These equilibrium values, however, may be either fairly stable along successive generations or, on the contrary, more or less strongly oscillatory, with a characteristic pattern of oscillations, as exemplified in Fig 1 (computed using the extended model). The - self-generated - oscillatory pattern, when it occurs, is characterized by a (sub-) constant period as well as a (sub-) constant shape of oscillations, usually with a very sharp decreasing rate and a 
comparatively slower increasing rate, especially as regards the host-oscillations (Figs 1 and 2). In addition, the oscillations of the host and of the parasite densities have (of course) the same periodicity, with the parasite oscillations being, yet, slightly out of phase (slightly delayed) as compared to the host oscillations (Figs. 1 and 2).

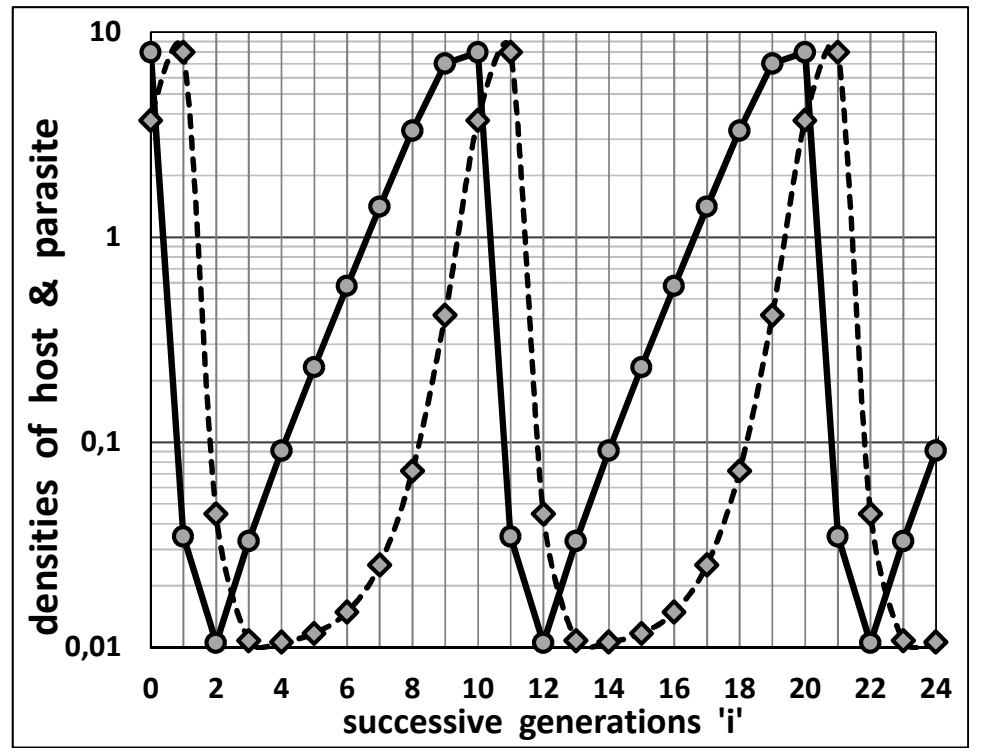

Fig. 1. A computed scenario of oscillatory equilibrium in host-parasite dynamics, with a predicted 10 years cyclic periodicity, according to the chosen parameters: $K=100, r=2.5$, $a=6.0, f=0.3, v=1, e_{H}=e_{P}=0.01$

Discs: density of the host species; diamonds: density of the parasite species. Notice the logarithmic scale for densities of occurrence of host and parasite

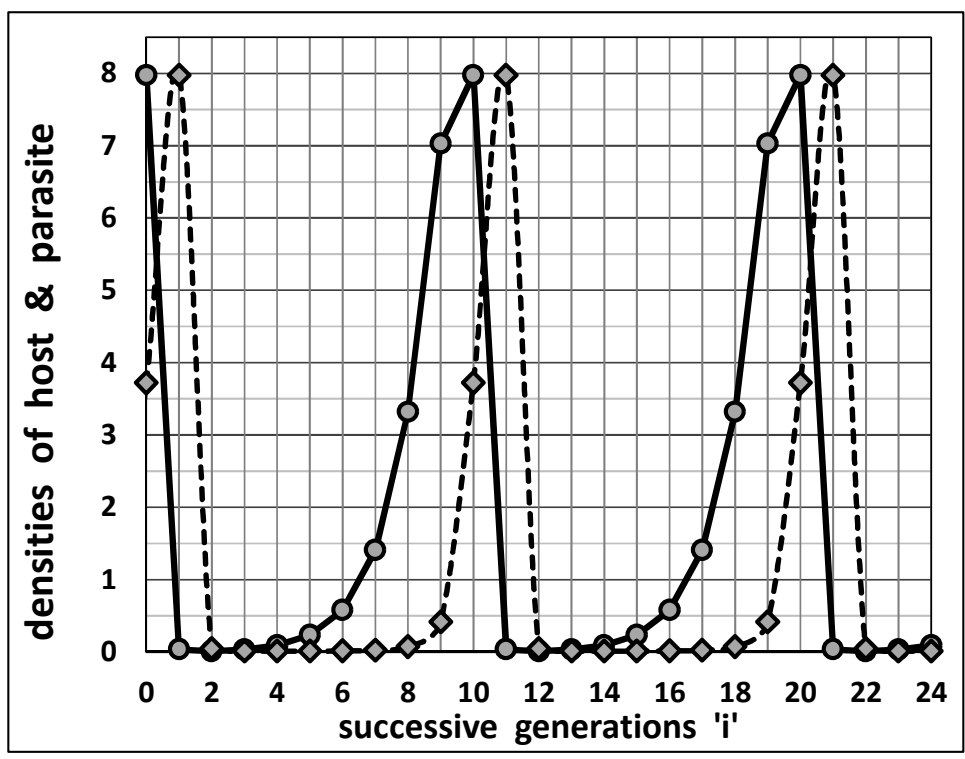

Fig. 2. Same as Fig. 1. with natural scale for densities - providing a more realistic, immediate appreciation of the amplitude of the variations of the densities of the host and the parasite along their respective cyclic (here decennial) oscillations

Here, roughly, two years of high abundances, two years of medium abundances and six years of rarity or virtual absence 
The occurrence of either stable or oscillatory regimes is dependent on the values taken by each of the seven main driving parameters of population dynamics cited above: v', 'f', 'a' for the inherent rate of growth of the parasite; ' $r$ ' for the inherent rate of growth of the host; ' $K$ ' accounting for the usually limited resource available for the host; ' $e_{H}$ ' and ' $e_{p}$ ' resulting from the metapopulation context. The respective influences, in this respect, of ' $a$ ', ' $r$ ', ' $K$ ', ' $e_{H}$ ' and ' $e_{P}$ ' will be briefly illustrated hereafter.

\section{INFLUENCES OF THE MAIN DRIVING PARAMETERS ON HOST \& PARASITE DENSITIES}

\subsection{Influence of the Level of Inherent Rate of Increase of the Parasite Species}

With increasing reproductive performance of the parasite (here, for example, improved foraging efficiency 'a' of the ovipositing females of the parasite), the pattern of variations of the host (and of the parasite) density along successive generations goes through three different phases of dynamic equilibrium, labelled respectively $S_{1}$, Os, $\mathrm{S}_{2}$ (Figs. 3 and 4):

(i) A phase " $S_{1}$ " of stable equilibria, where the host density is at a high level, yet monotonically decreasing with increasing parasite reproductive performance, while the parasite density increases monotonically and rather drastically - from its low, original level;

(ii) A phase "Os" of oscillating equilibria, with a strong unimodal variation in the amplitudes $\left[\mathrm{h}_{\max }-\mathrm{h}_{\min }\right]$ and $\left[\mathrm{p}_{\max }-\mathrm{p}_{\min }\right]$ of the oscillating densities of both the host and the parasite respectively, and a parallel (but comparatively slight) unimodal variation in the period length of the oscillation cycles (mean duration of one cycle);

(iii) A second phase " $\mathrm{S}_{2}$ " of stable equilibria, where not only the (low) density of the host but also the (low) density of the parasite both decrease monotonically with increasing own reproductive performance of the parasite.

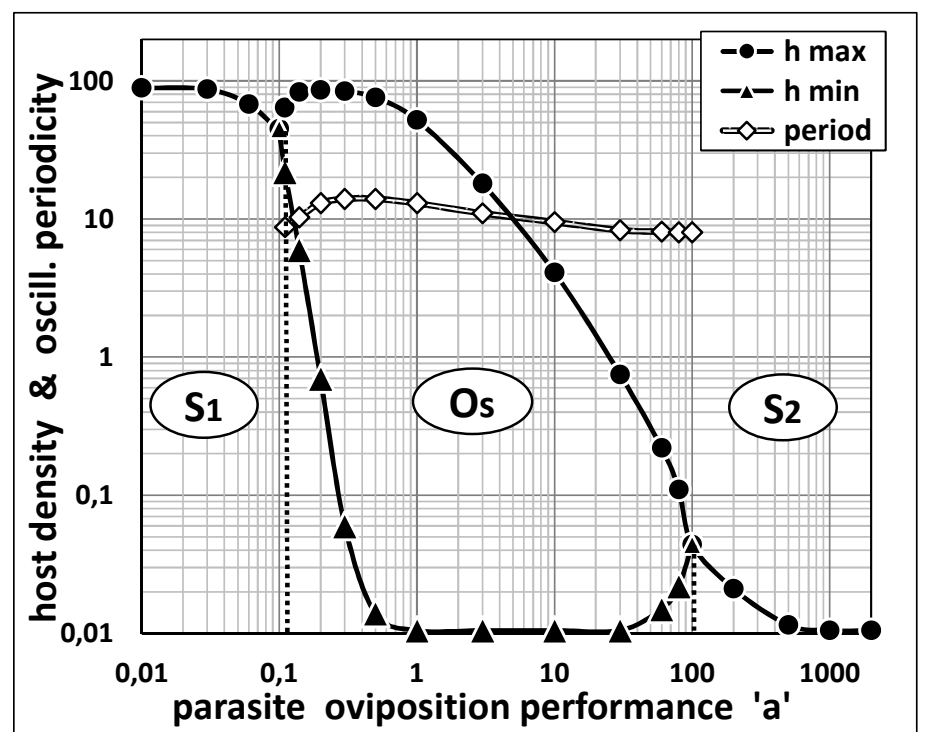

Fig. 3. Host density plotted against increasing reproductive performance of the parasite (here the foraging performance ' $a$ ' of ovipositing females) - all other driving parameters of the interaction remaining unchanged. Typical display of three equilibrium phases $-S_{1}$, Os, $S_{2}-$ in the interactive dynamics of the host-parasite system. $S_{1}$ and $S_{2}$ are the two phases where the densities of the host and the parasite both reach stable levels of their respective densities, while Os is the phase of oscillatory equilibrium for both host and parasite densities, as exemplified in Figs. 1 and 2

Discs: density of the host: (i) in the stable equilibrium phases and (ii) at the maximum (peak) density along the oscillatory phase. Triangles: lower (trough) density of the host along the oscillatory phase. Diamonds: period length of a cycle in the oscillatory phase, in term of number of generations.

$\left[K=100, r=2.5, a=\right.$ variable $\left., f=0.3, v=1, e_{H}=e_{P}=0.01\right]$ 


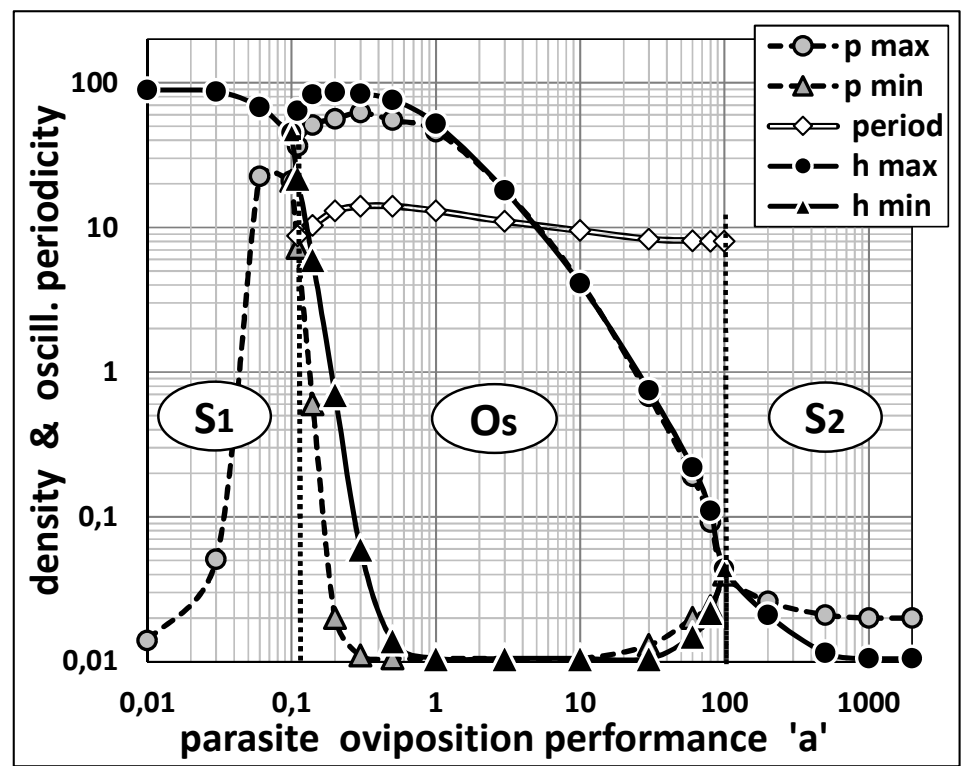

Fig. 4. Both host and parasite densities plotted against increasing reproductive performance (foraging efficiency ' $a$ ') of the parasite, all other driving parameters of the interaction remaining unchanged, in particular $r=2.5$

Discs: densities of the host (black) and the parasite (grey) in the stable equilibrium phases and at the maximum (peak) density along the oscillatory phase. Triangles: lower (trough) densities of the host (black) and the parasite

(grey) along the oscillatory phase. Diamonds: period length of a cycle along the oscillatory phase, in term of number of generations. [ $K=100, r=2.5, a=$ variable, $\left.f=0.3, v=1, e_{H}=e_{P}=0.01\right]$

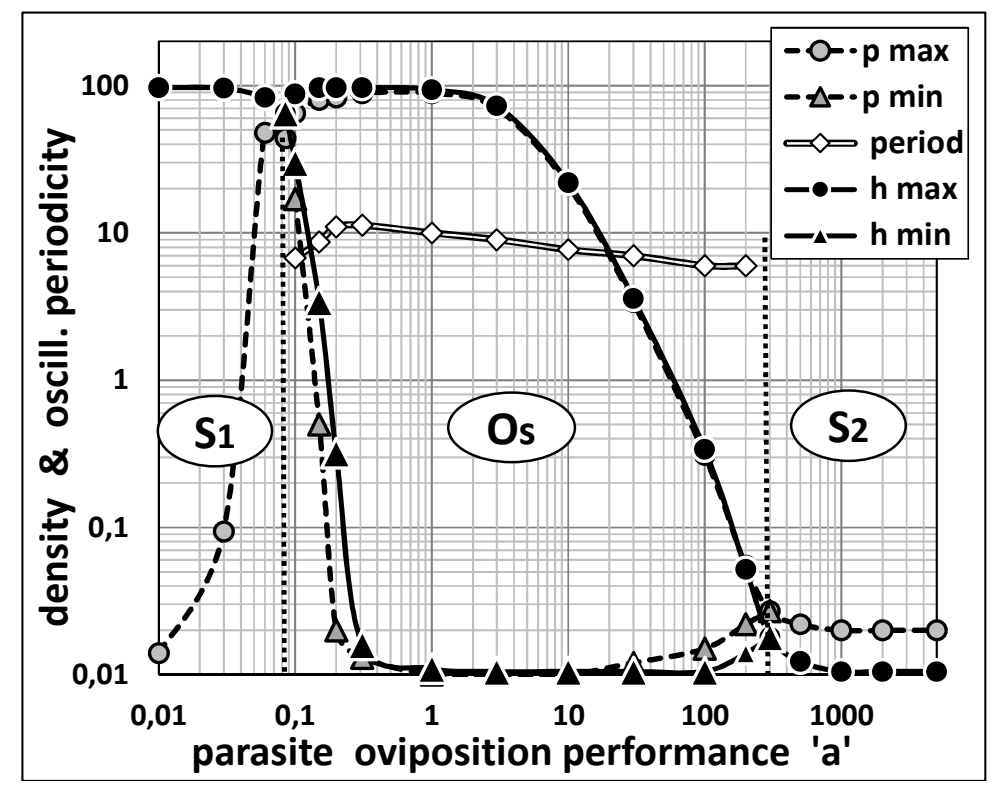

Fig. 5. Both host and parasite densities plotted against increasing reproductive performance (foraging efficiency ' $a$ ') of the parasite, all other driving parameters of the interaction remaining unchanged, in particular $r=5.0$

Discs: densities of the host (black) and the parasite (grey) in the stable equilibrium phases and at the maximum (peak) density along the oscillatory phase. Triangles: lower (trough) densities of the host (black) and the parasite (grey) along the oscillatory phase. Diamonds: period of cycle along the oscillatory phase, in term of number of generations. $\left[K=100, r=5.0, a=\right.$ variable, $\left.f=0.3, v=1, e_{H}=e_{P}=0.01\right]$ 
Thus, the same succession of three regimes of dynamic equilibrium stands for the variations of the parasite density as well as for the host density, along successive generations, as clearly demonstrated in Figs 4 and 5. And, as expected, this succession (in particular the onset and the final vanishment of the oscillatory regime) is in strict coordination between the host and the parasite. And this, because the stable, as well as the cyclic, dynamic equilibria depend on mutually linked, retroacting mechanisms between the host and the parasite.

This overall pattern of succession, $\mathrm{S}_{1} \rightarrow$ Os $\rightarrow$ $\mathrm{S}_{2}$, along steadily increasing levels of the reproductive efficiency of the parasite, looks quite robust, remaining qualitatively unaffected by variations of the other parameters, ' $r$ ', ' $K$ ' and the immigration rates ' $\mathrm{e}_{H}$ ', ' $\mathrm{e}_{\mathrm{p}}$ '. For example, Fig. 5 provides the sketch computed for a different inherent rate of growth ' $r$ ' of the host population: $r$ $=5$ in Fig. 5 , instead of $r=2.5$ in Figs. 3 and 4 .

Going in more details, regarding the specifically interesting cyclic regime, it appears that the amplitude of a cycle (the gap between the maximum and the minimum values of host as well as parasite densities) and its periodicity (period length of a cycle) both have unimodal responses to the increasing reproductive efficiency of the parasite, all across the oscillatory regime: both the amplitude and the period of cycles quickly increase at first, pass by a maximum and then progressively decrease. The magnitude of variation in amplitudes is obviously incommensurate with the magnitude of variation in cycle period length but, nevertheless, both amplitude and period length look fairly well correlated (Fig. 6).

The possible adaptative significance of the onset and the further long persistence of the oscillatory regime of host and parasite densities spontaneously inserting itself between the two stable regimes $S_{1}, S_{2}$ - is tentatively interpreted later, in the Discussion section.

\subsection{Influence of the Level of the Inherent Rate of Increase of the Host Species}

As it was the case for the parasite, increasing the reproductive performance (inherent growth rate ' $r$ ') of the host begins with a stable equilibrium regime, S1, for the densities of both the host and the parasite, next followed by the onset of an oscillatory (cyclic) regime, Os, when a critical value of ' $r$ ' is reached and then exceeded: Fig. 7.

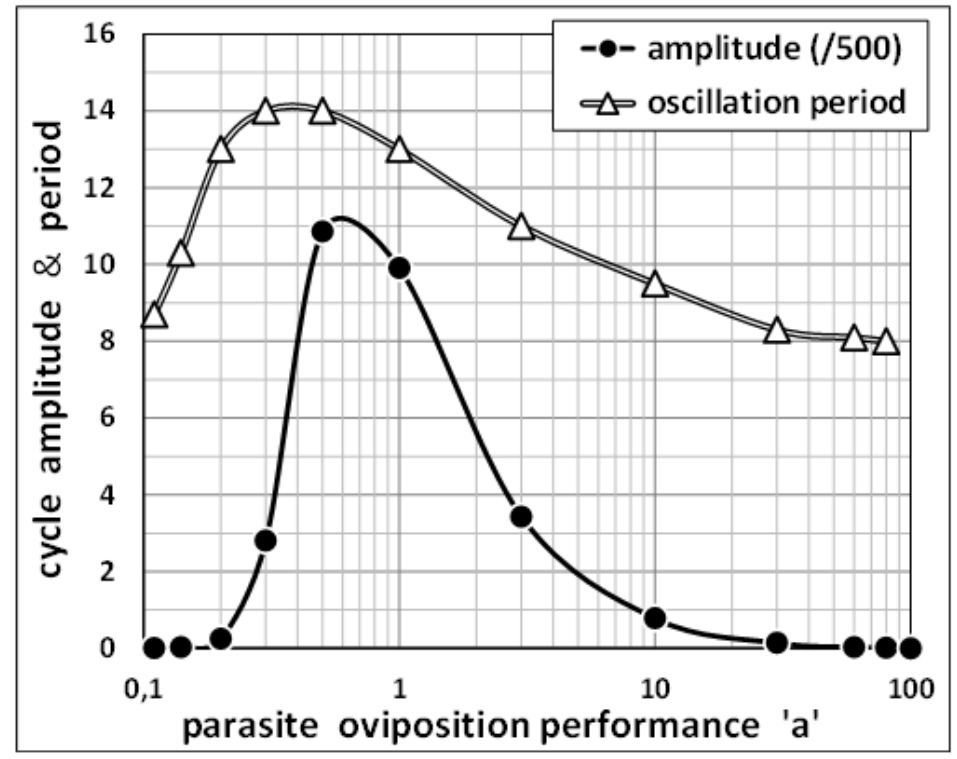

Fig. 6. Amplitudes of variation of (i) the host density (gap between peak and trough densities along a cycle) and (ii) the period length (i.e. the duration of a cycle, in term of number of successive generations) for the cyclic regime, plotted against increasing reproductive performance (foraging efficiency ' $a$ ') of the parasite, all other driving parameters of the interaction remaining unchanged. Note that cycle amplitude has been divided by 500.

$\left[K=100, r=2.5, a=\right.$ variable, $\left.f=0.3, v=1, e_{H}=e_{P}=0.01\right]$ 


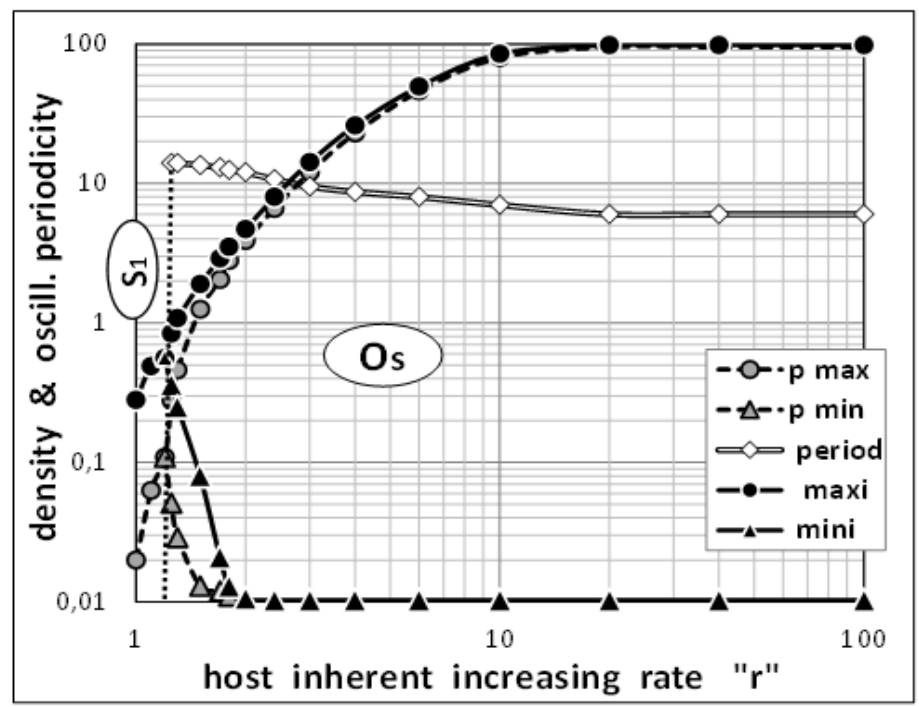

Fig. 7. Both host and parasite densities plotted against increasing inherent growth rate ' $r$ ' of the host, all other driving parameters of the interaction remaining unchanged

Discs: densities of the host (black) and the parasite (grey) in the stable equilibrium phases and at the maximum (peak) density along the oscillatory phase. Triangles: lower (trough) densities of the host (black) and the parasite

(grey) along the oscillatory phase. Diamonds: period of cycle along the oscillatory phase, in term of number of generations. [K=100, $r=$ variable, $\left.a=6, f=0.3, v=1, e_{H}=e_{P}=0.01\right]$

And here also, and once again, the patterns for host and parasite are in strict coordination. But, contrary to the case above, the cyclic regime is further maintained whatever the value of ' $r$ '; that is, no second stable equilibrium pattern is ever reached. Also, the amplitude and the period length of cyclic densities, are negatively correlated (instead of positively above): thus, while the period of cycle is steadily decreasing at first (and finally stabilizing) with larger reproductive performance of the host, the amplitude of cycles, on the contrary, is quickly and strongly increasing at first (then stabilizing).

\subsection{Influence of the Carrying Capacity (Resource Availability for the Host)}

As might have been expected, increasing the level of available resource for the host (i.e. increasing the carrying capacity ' $K$ '), leads to a pattern (Fig. 8) which is somewhat similar to what was observed above when improving the inherent growth rate ' $r$ ' of the host. All the remarks made above (as regards the influence of the inherent growth rate of the host) apply, here, with the exception of a now positive correlation between the amplitude and the period of the cyclic densities. In particular, the cyclic regime is maintained whatever the value of ' $\mathrm{K}$ '; that is, no second stable equilibrium is ever reached.

\subsection{Influence of the Immigration Rate of the Parasite}

Fig. 9 provides an account of the variations of the host density with increasing level of the parasite immigration ' $\mathrm{e}_{\mathrm{p}}$ ', all other parameters being unchanged. Increasing the level of parasite immigration should, expectedly, have an effect on the variations of host density being somehow reminiscent of what is observed with growing reproductive efficiency ' $a$ ' of the parasite. Hence, the qualitative similarity that can be recognized between the right part of Fig. 3 (for values of ' $a$ ' larger than 6.0 , as chosen in Fig. 9) and the right part of Fig. 9 (when ' $e_{p}$ ' comes in excess of the value, $e_{p}=0.01$ chosen in Fig. 3). Here also, a positive correlation between the amplitude and the period of the cyclic densities is highlighted. The consistent decrease of the period length with increasing levels of parasite immigration is best shown in Fig. 10, with untransformed coordinates.

\subsection{Influence of the Immigration Rate of the Host}

Fig. 11 provides an account of the variations of the host density with increasing levels of immigration ' $\mathrm{e}_{\mathrm{H}}$ ' of the host itself, all other 
parameters being unchanged. Here also (as for the influence of the parasite immigration rate), increasing the level of host immigration rate should, conceivably, have an effect on the variations of host density being somehow reminiscent of what is observed with growing inherent rate of growth ' $r$ ' of the host itself (as it is shown in Fig. 7).

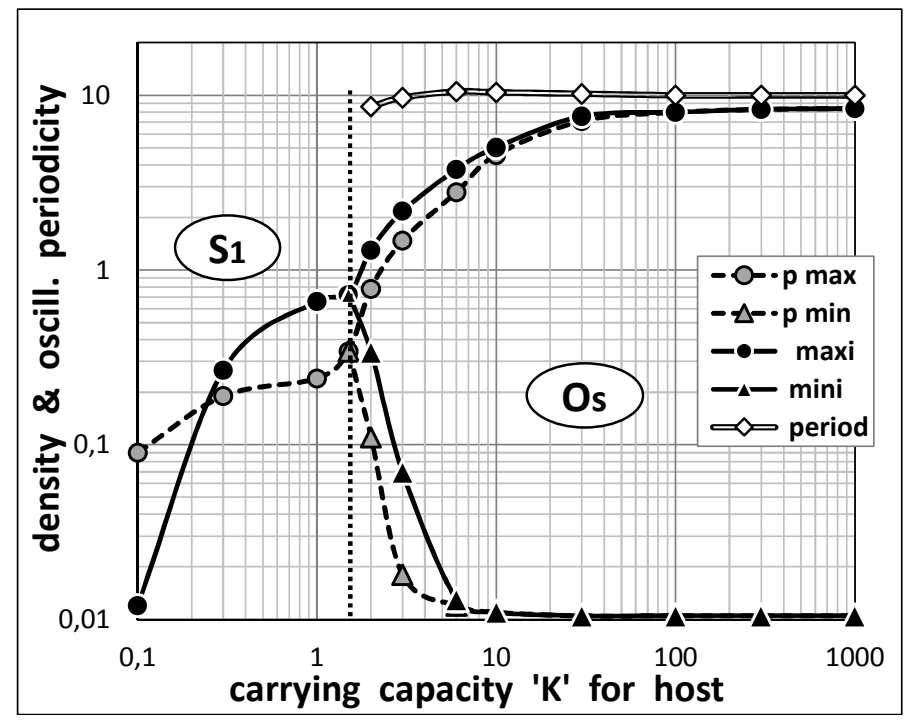

Fig. 8. Both host and parasite densities plotted against increasing carrying capacity ' $\mathrm{K}$ ' for the host, all other driving parameters of the interaction remaining unchanged

Discs: densities of the host (black) and the parasite (grey) in the stable equilibrium phase and at the maximum (peak) density along the oscillatory phase. Triangles: lower (trough) densities of the host (black) and the parasite (grey) along the oscillatory phase. Diamonds: period of cycle along the oscillatory phase, in term of number of generations. [K: variable, $\left.r=2.5, a=6, f=0.3, v=1, e_{H}=e_{P}=0.01\right]$

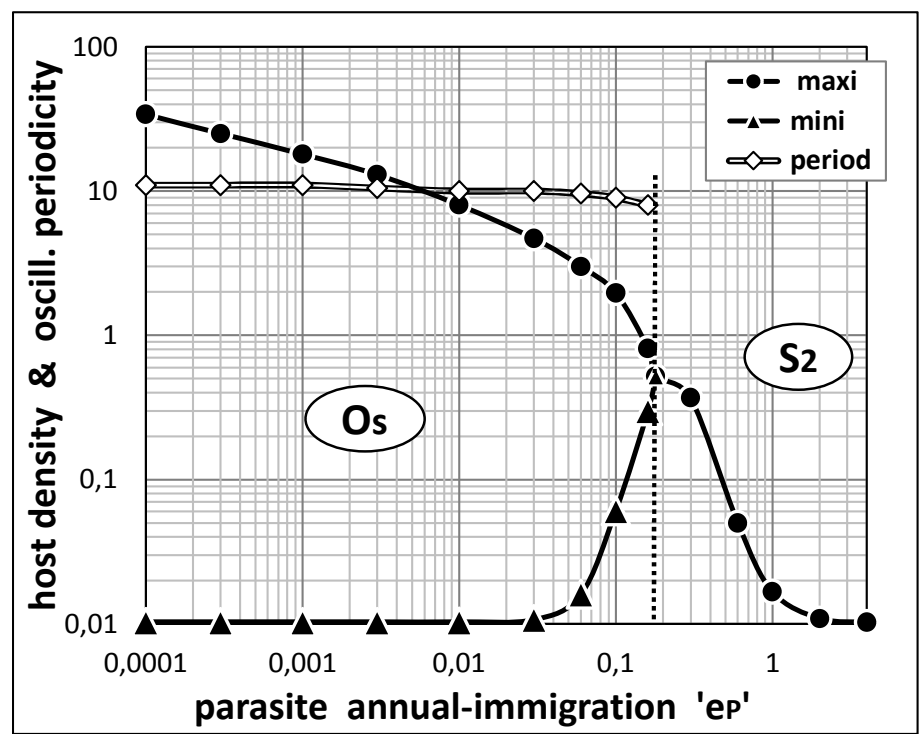

Fig. 9. Host density plotted against increasing immigration rate ' $e_{\mathrm{P}}$ ' of the parasite, all other driving parameters of the interaction remaining unchanged

Discs: density of the host in the stable equilibrium phase and at the maximum (peak) density along the oscillatory phase. Triangles: lower (trough) densities of the host (black) and the parasite (grey) along the oscillatory phase. Diamonds: period of cycle along the oscillatory phase, in term of number of generations.

$$
\left[K=100, r=2.5, a=6, f=0.3, v=1, e_{H}=0.01, e_{P} \text { variable }\right]
$$




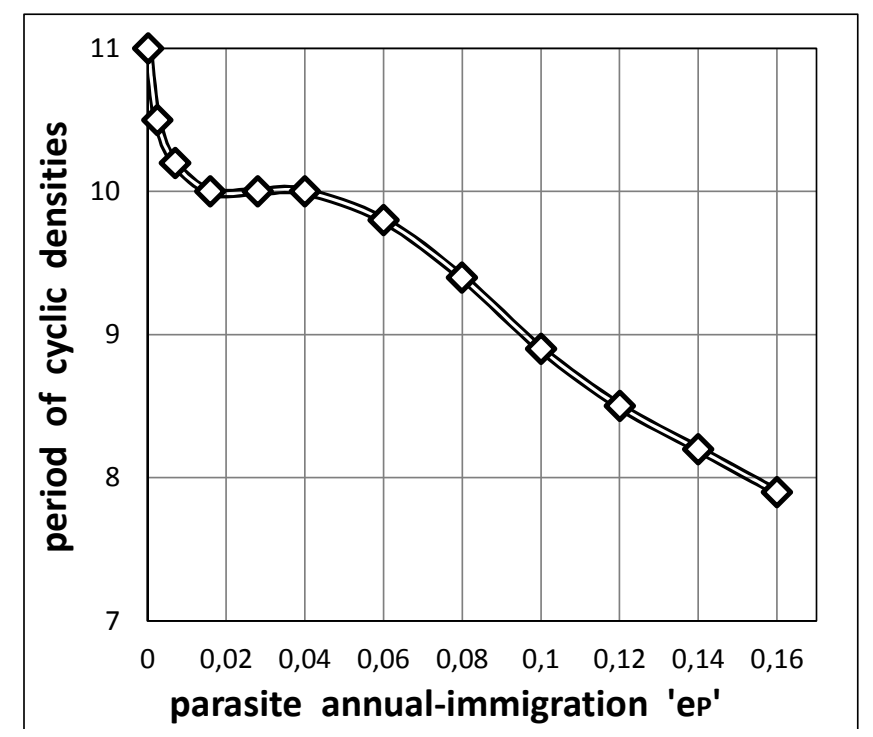

Fig. 10. Focus on the influence of the immigration rate ' $e_{p}$ ' of the parasite on the period length of cyclic densities of both the host and the parasite, in term of number of generations

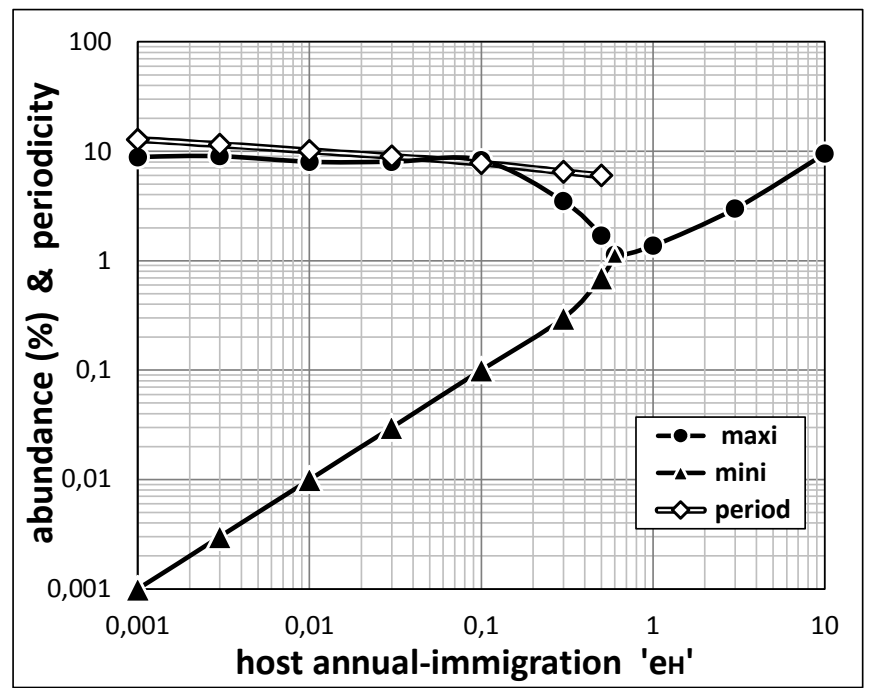

Fig. 11. Host density plotted against increasing immigration rate ' $e_{H}$ ' of the host itself, all other driving parameters of the interaction remaining unchanged

Discs: density of the host in the stable equilibrium phase and at the maximum (peak) density along the oscillatory phase. Triangles: lower (trough) densities of the host (black) and the parasite (grey) along the oscillatory phase. Diamonds: period of cycle along the oscillatory phase, in term of number of generations.

$$
\left[K=100, r=2.5, a=6, f=0.3, v=1, e_{P}=0.01, e_{H} \text { variable }\right]
$$

But, here, in addition, the rate of host immigration constrains, of course, the minimum value of host density being at least equal to ' $e_{H}$ '. Hence the imposed sub-linear increase of the minimum value of host density (Fig. 11), which is not the case with growing inherent rate of growth ' $r$ ' of host alone (Fig. 7). As a consequence, the minimum density ends up joining the maximum density, thus finally putting an end to the oscillatory regime (Fig. 11). While, on the contrary, the oscillatory regime is consistently maintained unending with growing inherent rate of growth ' $r$ ' (Fig. 7). A still positive correlation between the amplitude and the period of the cyclic densities is highlighted. The consistent decrease of the period length with increasing levels of host immigration is best shown at Fig. 12 with untransformed coordinates. 
4.6 A Synthetic Overview of the Influences of the Five Main Driving Parameters on the Amplitude and the Period Length of Oscillations of Densities in the Cyclic Regime

Table 1 provides a schematic summary of the trends of variations of the amplitude and the period length of oscillations of both the host and the parasite densities with increasing values of the five major governing factors of host-parasite population dynamics, as synthetized from the preceding results.

\section{EXAMPLE OF APPLICATION:} INTERPRETING THE ROLE OF THE METAPOPULATION CONTEXT ON THE PERIOD LENGTH OF OUTBREAKS OF THE PINE PROCESSIONARY MOTH

Cyclic patterns in the population dynamics of forest moth defoliators have been frequently reported [4-6, 10-15]. In particular, populations of the Pine processionary moth, Thaumetopoea pityocampa (Denis \& Schiffermüller), are usually subjected to more or less strong and regular outbreaks which are of major concern for both forest and human health [11]. In a broad scale study encompassing the whole of impacted regions in France, LI et al. [10] report on a decrease (highly significant, $p=0.0009$ ) of the length of the period separating successive outbreak picks of the pine processionary moth when the pine cover is increasing in the surrounding landscape. That is, the period length consistently decreases when infested stands are obviously less isolated. This suggests, in turn, that the reduction of the average length of periodic cycles of density may ultimately result from an increasing rate of immigration of the moths and the parasites as a consequence of stands being less isolated.

This expectation is actually supported, and thus satisfactorily explained, by the extended model, as already suggested above. Thus, Fig. 13 highlights a consistent trend for a steadily decrease of the period length of the cyclic oscillations of both host and parasite densities when immigration rates of the host and the parasite increase, all other factors being unchanged. The trend looks robust, being maintained for a series of different values of the other main driving parameters of host-parasite dynamics (Fig. 13).

Moreover, the trend still holds true when only the immigration rate of the host is increasing alone. Incidentally, the model predicts that, as is the case for the period length, the amplitude of the cyclic oscillations should also decrease with increasing immigration rates, due to both the decrease of the peak density and the strong increase of the density trough (unpublished results).

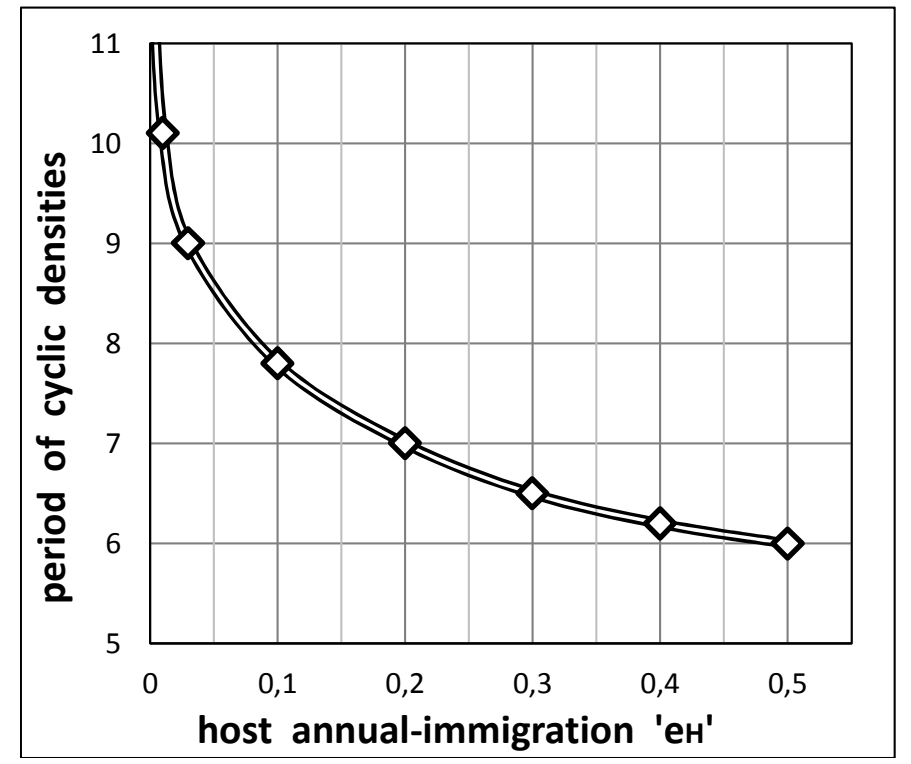

Fig. 12. Focus on the influence of the immigration rate ' $e_{\mathrm{H}}$ ' of the host on the period length of cyclic densities of both host and parasite, in term of number of generations 
Table 1. General trends of variations of the amplitude and the period length of the cyclic regime of host-parasite densities, as a function of increasing values of (i) the reproductive efficiency of the parasite, (ii) the reproductive efficiency of the host, (iii) the available resource for the host, (iv) the immigration rate of the parasite, (v) the immigration rate of the host

\begin{tabular}{llllll}
\hline Cyclicity & $\begin{array}{l}\lambda \text { reprod } \\
\text { parasite }\end{array}$ & $\begin{array}{l}\lambda \text { reprod } \\
\text { host }\end{array}$ & $\begin{array}{l}\lambda \text { resource } \\
\text { for host }\end{array}$ & $\begin{array}{l}\lambda \text { immigr } \\
\text { parasite }\end{array}$ & $\begin{array}{l}\lambda \text { immigr } \\
\text { host }\end{array}$ \\
\hline amplitude & $\lambda \searrow$ & $\lambda \rightarrow$ & $\lambda \rightarrow$ & $\searrow$ & $\searrow$ \\
period length & $\lambda \searrow$ & $\searrow \rightarrow$ & $\lambda \rightarrow$ & $\searrow$ & $\searrow$ \\
\hline
\end{tabular}

Trends are computed using the extended version of the Nicholson \& Bailey model

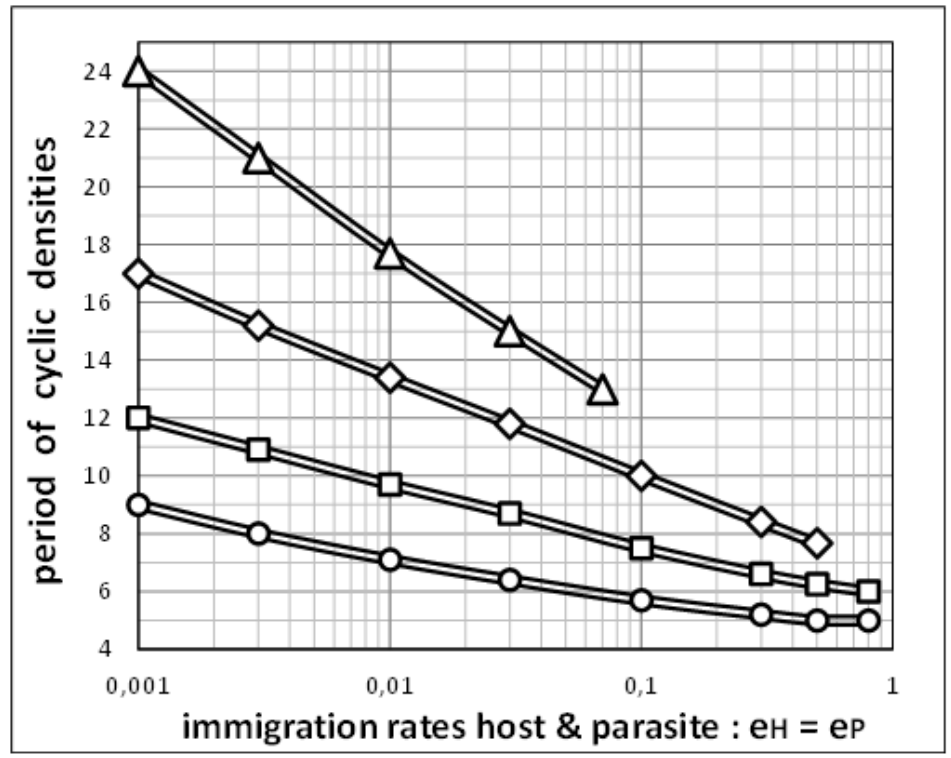

Fig. 13. Computed period length of outbreaks (cyclic regime of densities) plotted against increasing immigration rates of both the host and the parasite (assumed being equal: $e_{H}=e_{P}$ ), all other driving parameters of the interaction remaining unchanged

Triangles: $r=1.3, a=1.0$; Diamonds: $r=2.3, a=1.0$; Squares: $r=4.0, a=2.0$; Discs: $r=10.0, a=4.0$.

$$
\left[K=100, f=0.3, v=1, e_{H}=e_{P}: \text { variable }\right]
$$

\section{DISCUSSION}

Host-parasite interactions - and their outcome in term of population dynamics - certainly involve too many different causes to be always appropriately described in sufficient details by comparatively simple models such as the classical Nicholson \& Bailey model or, even, the extended version implemented here. For example, CLARK [16] (reviewed in [5]) provides a good illustration of the multiplicity of biotic and abiotic factors jointly involved in the very irregular population dynamics of the psyllid Cardiaspina albitextura.

Yet, on the other hand, cases are not so rare, where the population dynamics turns out not to be so irregular and, therefore more amenable to handy modelling. Admittedly, even so, such simple models as Nicholson \& Bailey's and its extended version, could still provide insufficiently accurate and reliable accounts of the detailed variations of host and parasite abundances. But, at least, the major trends of the host-parasite mutual dynamics - and their associated rational explanations - can be efficiently derived from such models, and this was, indeed, the primary focus of the present study which aims, at first, to provide some educational survey of the question. In particular, specific attention was devoted to the prominent question of the conditions triggering the onset of cyclic oscillations of host and parasite densities (Figs 1 and 2) and the frequent outcome of cyclicity in terms of more or less dramatic outbreaks of forest defoliators - as this is of both speculative and practical interest. 


\subsection{Implementing Rational Scenarios to Capture Some Main Drivers of Host- Parasite Dynamics and to Tentatively Provide Rational Interpretations}

Among the major ascribable causes, the respective influences of the inherent rates of increase of either the host or the parasite have been re-examined, here, and were given a presumably original synthetic presentation (Figs. 3 to 7), hopefully consistent with an educational goal (of course, this first step was already accounted for, since a while, by the original Nicholson \& Bailey model).

With the same concerns, the influences of other major factors, specifically addressed in the extended version of the Nicholson \& Bailey developed for this purpose, are subsequently considered, namely: the influence of the level of available resource for the host (Fig. 8) and the respective roles of the immigration rates of either the parasite (Figs. $9 \& 10$ ), or the host (Figs. 11 $\& 12$ ), or of both partners (Fig. 13).

All together, these Figs. - and the synthetic presentation summarized in Table 1, derived from these Figs - aim at displaying a panorama of how and for which reasons, the pattern 'Os' of sharp oscillations of densities of both the host and the parasite may emerge from the backdrop of the otherwise stable dynamic equilibrium patterns, ' $S_{1}$ ' and ' $S_{2}$ '. And, in addition, how and for which reasons, both the amplitude and the periodicity of this sharp oscillations can vary in considerable proportions (occasionally leading to severe outbreaks of major practical concern).

Once again, these scenarios do not aim at providing accurate quantitative predictions, in terms of variations of host and parasite densities, under specific values of the main governing parameters (inherent rates of increase, available resource for the host, immigration rates). In fact, trying to capture and tentatively highlight at least the major trends, in a semi quantitative approach, is the only, but already significant ambition of the present extended model. Moreover, following on from this perspective, a second objective was to offer a tool for putting this approach into free practice. Thus, multiple scenarios, computed with the extended model (detailed in Appendix) and based on freely varied values of governing parameters, might well encourage some enlightening, fruitful exercises.

As an example of such "explorative scenarios", a theoretical argumentation has been put forward
(Fig. 13) to explain the observations reported by LI et al. [10], according to which a significant shortening in the outbreaks period length of the pine processionary moth was empirically associated to an increasing pine cover in the surrounding landscape.

Another example of meaningful scenario, not addressed here, but which would deserve further investigation, would be to proportionate the immigration rates of the host and of the parasite (at any given generation) to the corresponding densities of the host and the parasite at the preceding generation (instead of immigration rates remaining constant along successive generations), as this would more closely approach reality.

Now, beyond, the main practical concern of seeking for rational explanations to be given to reported field observations, some more speculative aspects of the host-parasite dynamics can also be addressed and discussed hereafter.

\subsection{A Possible "Adaptive" Role for the Onset of Cyclicity in Host-parasite Systems?}

Let examine more deeply what happens when the parasite reproductive efficiency (for example, the mean foraging area 'a' of ovipositing females) is steadily increasing (all other parameters being unchanged), as considered in Figs. 3 and 4.

* stable equilibrium phase $\mathrm{S}_{1}$ (extending up to 'a' $=0.1$ ) : at first, with very low values of parasite foraging area (here, for ' $a$ ' $<0.03$ ) the density of the parasite slowly increases but remains of course very weak, while the density of the host remains very high, not far from the carrying capacity $\mathrm{K}$ for the host. Then (here, for ' $\mathrm{a}$ ' > 0.03), the density of the parasite increases drastically while, accordingly, the density of the host begins slowly decreasing. Extrapolating this trend would promptly lead the parasite density to rejoin and then strongly overstep the density of the host. The latter would, therefore, be rapidly doomed to an ultimate collapse. But, precisely, entering the oscillatory pattern will efficiently challenge this trend!

* oscillatory (cyclic) equilibrium phase Os (here for $0.1<$ 'a' < 100) : entering the oscillatory pattern obviously opposes with strong efficacy the extrapolated trend for the parasite density to overstep the density of the host, thereby 
preventing the, otherwise, quick and inevitable collapse of the host density. And all along the stage of cyclic equilibrium, the extrapolated trend for the parasite density to strongly exceed the density of the host is clearly dismissed, despite the increase by a factor 1000 of the foraging area 'a' ('a' increasing from 0.1 to 100 across the oscillating phase, see Fig. 14). And, thus, despite the corresponding increase in the reproductive efficiency of the parasite.

* stable equilibrium phase $\mathrm{S}_{2}$ : eventually, when the efficiency of the parasite finally overcomes the "counteroffensive" (the "buffering" effect) of the oscillatory process (here for parasite foraging area ' $a$ ' > 100), then, the parasite density henceforward markedly oversteps the density of the host. But this, indeed, can be considered a "Pyrrhic victory", since the parasite supremacy over the density of its host is a supremacy obtained at the price of a now quite low absolute levels of densities, not only for the host, but for the parasite as well !

This, indeed, is still better characterized in quantitative terms by precisely considering the variation of the ratio $\pi$ of the parasite average density to the host average density, i.e. the "parasitism pressure" $\pi$. The buffering effect on the parasitism pressure, resulting from the onset of the cyclic regime Os, is clearly highlighted in Fig. 14. Moreover, the implemented model shows a trend for this buffering effect going stronger and extending further with increasing inherent growth rate ' $r$ ' of the host species, thereby suggesting that it is the "reactivity" of the host species which features instrumental in this respect. And, indeed, this buffering effect looks as if it was at the "benefit" of the host. Thus, for these reasons, the onset of the cyclic regime might relevantly be considered "adaptative", from the point of view of the host own success.

Thus, as a whole, the oscillatory process (i) opportunely establishes itself when the drastically increasing density of the parasite dangerously approaches the level of the decreasing density of the host, and then, (ii) lengthily makes opposition to the trend for the parasite to overrun the host density - a process which may arguably be considered as having a truly efficient buffering influence on the severity of parasitism outcome and finally strongly postpones the ultimate success of the parasite (i.e. parasite density overstepping host density: $\pi$ > 1). In this respect, the cyclicity of both host and parasite densities may appropriately be viewed as a kind of "relaxing oscillations".

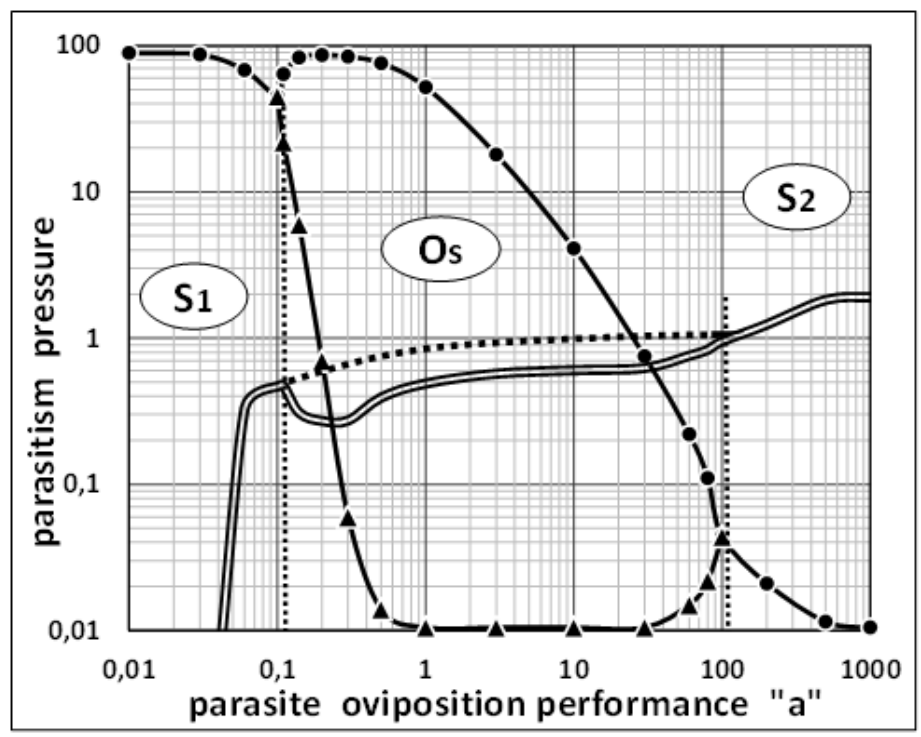

Fig. 14. The same scenario as in Fig. 3 with, superimposed, the relative parasitism pressure, quantified in term of the computed ratio $\pi$ of the average parasite density to the average host density (coarse double line)

Note the sudden drop of $\pi$ at the onset of the oscillating regime $\left(S_{1} \rightarrow O s\right)$. The coarse dotted line stands for the hypothetic value of $\pi$ if the oscillatory phase would not exist (hand drawing). Thus, the gap between the dotted line and the double line highlights the "buffering" effect exerted on the parasitism pressure $\pi$, presumably attributed to the cyclic (oscillating) regime. [ $K=100, r=2.5, a=$ variable, $\left.f=0.3, v=1, e_{H}=e_{P}=0.01\right]$ 
Thus, the cyclicity of both the host and the parasite densities may well be considered being "adaptive" not only, immediately, for the host only, but also for the host-parasite system as a whole, as far as it favors and extends the coexistence between two (or, more largely, several) species involved in multi-trophic relationships. Multi-trophic relationships which, indeed, make the generality of structuring processes shaping ecosystems worldwide. Reasoning this way thus uncovers the (likely unexpected) fundamental importance of this auto-adaptative mechanism, where a strongly oscillating regime of species densities ultimately contributes to avoid the catastrophic extinction of some species and, thereby, promotes a less unstable co-existence among species, within species-rich ecosystems.

\section{CONCLUSION}

The extended version of the Nicholson \& Bailey model, implemented here, does not pretend to provide an accurate quantitative account of the host-parasite dynamics but rather aims at enlightening some major trends regarding the influence of five major drivers on the hostparasite dynamics in general, thereby helping to propose some likely rational interpretations of various aspects of these dynamics, as reported from the growing series of published field observations. Indeed, this approach, promoting the development of "not too complex models", is in full line with the final recommendations by HUNTER \& DWYER [9]. An illustrative example was proposed, tentatively providing a rational explanation of the influence of the metapopulation context on the period length (and the degree of severity) of the outbreaks of the pine processionary moth dynamics.

In quite a more speculative approach, the implementation of this extended model may serve, as well, as a convenient tool (i) for elaborating scenarios involving various combinations of values of the five drivers and, thereby, (ii) for testing a series of conceptual hypothesis on the possible role of these combined factors on the host-parasite dynamics, either quasi-stable dynamic equilibrium or strongly oscillating regime.

\section{ACKNOWLEDGEMENTS}

I acknowledge the fruitful comments from two anonymous Reviewers, based on the original manuscript.

\section{COMPETING INTERESTS}

Author has declared that no competing interests exist.

\section{REFERENCES}

1. Elton C. Periodic fluctuations in the number of animals: their causes and effects. Journal of Experimental Biology. 1924;2:119-163.

2. Mac Lulich DA. Fluctuations in the numbers of the varying hare (Lepus americanus). University Toronto Stud. Biol. Serv. 1937;43:1-136.

3. Elton $\mathrm{C}$, Nicholson $\mathrm{M}$. The ten-year cycle in numbers of the Lynx in Canada. Journal of Animal Ecology. 1942;11:215-244.

4. Varley GC. Population changes in German forest pests. Journal of Animal Ecology. 1949;18:117-122.

5. Dajoz R. Dynamique des Populations. Masson, Paris; 1974.

6. Barbault R. Ecologie des populations et des peuplements. 198; Masson, Paris.

7. Myers JH, Cory JS. Population cycles in forest Lepidoptera revisited. Annual Review of Ecology, Evolution and Systematics. 2013;44:565-592.

8. Nicholson AJ, Bailey VA. The balance of animal populations. Proceedings of the Zoological Society of London. 1935;551598.

9. Hunter AF, Dwyer G. Outbreaks and interacting factors: insect population explosions synthetized and dissected. Integrative Biology. 1998;166-177.

10. Li S, Daudin JJ, Piou D, Robinet C, Jactel $H$. Periodicity and synchrony of pine processionary moth outbreaks in France. Forest Ecology and Management. 2015; 354:309- 317.

11. Abgrall JF, Bouhot L. Population fluctuations of the pine processionary moth (Thaumetopoea pityocampa Schiff.) in France from 1969 to 1989. In: Proceedings of the XIXth IUFRO World Congress. Montréal (Québec); 1990.

12. Parry D, Spence JR, Volney WJA. Responses of natural enemies to experimentally increased populations of the forest tent caterpillar, Malacosoma disstria. Ecological Entomology. 2003;22: 97-108.

13. Régnière J, Nealis VG. The fine-scale population dynamics of spruce budworm: survival of early instars related to forest 
condition. Ecological Entomology. 2008;33: 362-372.

14. Cory JS, Myers JH. Within and between population variation in disease resistance in cyclic population of western tent caterpillars: a test of the disease defense hypothesis. Journal of Animal Ecology. 2009;78:46-55.
15. Alfaro RI, Berg J, Axelson J. Periodicity of western spruce budworm in Southern British Columbia, Canada. Forest Ecology and Management. 2014;315:72-79.

16. Clark LR. The population dynamics of Cardiaspina albitextura (Psyllidae). Australian Journal of Zoology. 1964;12: 362-380.

\section{APPENDIX}

\section{Derivation of an Extended Formulation of the Nicholson \& Bailey Model}

\section{A.1- the classical Nicholson \& Bailey model}

The original Nicholson \& Bailey model accounts for the predator-prey interaction in discrete time and is more particularly (although not exclusively) designed to treat the parasite-host interaction in insects. Let ' $h h_{i}$ ' and ' $p_{i}$ ' be, respectively, the densities (numbers of individuals per unit area of investigation) of the host and of the parasite, at generation ' $i$ '. Besides - and in accordance with the Nicholson \& Bailey model - it is assumed that each parasite ovipositing female:

(i) is foraging at random for its selected kind of prey,

(ii) avoids oviposition upon an already egg-laid prey (or, if not so - which is likely - the second oviposition does not lead to viable offspring),

(iii) has some finite foraging area 'a' (also assuming that it is this foraging area, rather than the female's eggs load, which is the operational limiting factor in the parasite eggs deposition).

Also, the Nicholson \& Bailey model - as well as its present extension - relevantly apply if parasite and host share the same scale of successive generations, which indeed is often the case: [5]. Under these conditions, the predicted density of parasitized host individuals complies with a Poisson distribution $[5,9]$.

Accordingly, the densities $h_{i, \text { par }}$ and $h_{i, \text { non par }}$ of parasitized and non-parasitized hots are:

$$
\begin{aligned}
& h_{i, \text { par }}=h_{i} \cdot\left(1-\exp \left(- \text { a.f. } p_{i}\right)\right) \\
& h_{i, \text { non par }}=h_{i} \text {. } \exp \left(- \text { a.f. } p_{i}\right)
\end{aligned}
$$

with ' $f$ ' as the proportion of mated (and thus egg-laying) females within the whole population of parasites.

Let ' $r$ ' be the inherent rate of increase of the host (as it would be in the absence of parasitism and of any limitation in resource availability for the host development).

Accordingly, the density of hosts at generation $\mathrm{i}+1$ is:

$$
h_{i+1}=r \text {. } h_{i, \text { non par }}
$$

that is:

$$
h_{i+1}=r \cdot h_{i} \cdot \exp \left(- \text { a.f. } p_{i}\right)
$$

The density $p_{i+1}$ of parasites at generation $i+1$ is proportional to the density $h_{i, p a r}$ of parasitized hosts at generation $\mathrm{i}$ :

$$
\mathrm{p}_{\mathrm{i}+1}=\mathrm{v} .\left(\mathrm{h}_{\mathrm{i}, \mathrm{par}}\right)
$$


with ' $v$ ' as the average number of viable parasites individuals issued from each parasitized host individual (proportional, in particular, to the egg clutch-size laid by ovipositing female of the parasite, at the expense of a single host individual). The density of mated ovipositing females of the parasite at generation $i+1$ being, accordingly, equal to (f. $\left.p_{i+1}\right)$.

Accounting for the expression (1) of $h_{i, \text { par, }}$ it comes:

$$
p_{i+1}=v \cdot h_{i} \cdot\left(1-\exp \left(- \text { a.f. } p_{i}\right)\right)
$$

Note, incidentally, that equations (4) and (5) comply with the classical formalism of HASSELL: see equations (1) and (2) with $\mathrm{m}=0$ in reference [9].

\section{A.2- The extended Nicholson \& Bailey model involving both (i) the contribution of immigration- emigration and (ii) the limitation of available feeding resource for the host}

\section{A.2.1 Immigration-emigration}

Let $e_{H}$ and $e_{P}$ be the additional contributions (at each generation) of the balance between the immigration and the emigration of the host (resp. the parasite), in complement to the host and parasite densities computed above. It comes:

$$
\begin{aligned}
& h_{i+1}=r \cdot h_{i} \cdot \exp \left(- \text { a.f. } p_{i}\right)+e_{H} \\
& p_{i+1}=v \cdot h_{i} \cdot\left(1-\exp \left(- \text { a.f. } p_{i}\right)\right)+e_{P}
\end{aligned}
$$

\section{A.2.2 Limitation of available feeding resource for the host}

Moreover, let ' $R$ ' be the density of available feeding resource for the host insects (typically, for an herbivore insect, the density of leaves of the appropriate plant species, acceptable for the successful development of offspring) and ' $c$ ' the mean consummation of resource necessary to the full development of a host individual. Thus, the carrying capacity for the host insects (i.e. the density of host individuals that could be fed by the hypothetically exhaustive consummation of the available resource) is ' $K$ ', with:

$$
K=R / c
$$

The relationship between the densities of host individuals at the successive generations $i$ and $i+1$, provided by equation (4) (or equation (6) accounting for immigration-emigration), should then be modified to account for the limiting carrying capacity ' $K$ '.

The consequences of the limited availability - if any - of resource for the host depend, in particular, upon the pattern of intra-specific competition for this resource among co-occurring host individuals. Let consider successively two extreme hypotheses in this respect.

* according to a first - somewhat unrealistic - hypothesis, intra-specific competition for resource among co-occurring host individuals would be ideally avoided until the threshold of complete consummation of the entire available resource is finally reached. This would imply quite a strict regular pattern of distribution of eggs (and of the subsequent pattern of distribution of offspring) among the displayed resource, so as to prevent any scramble competition to occur at any time, until all available resource is actually consumed. The corresponding behavior of ovipositing host-females aiming at reducing at most the loss liable to intra-specific competition - would undoubtedly have some positive selective value per se. Yet, on the other hand, it would also require, as emphasized above, an accurate planning a priori of the spatial distribution of eggs among the displayed resource, based on a prior knowledge of the diet just necessary for each future larva. And this hypothetical behavior of ovipositing host-females would also involve, in turn, a significant cost devoted to the recognition and the avoidance of already egg-laid areas, as well as an extra time-expenditure when foraging for resource to comply efficiently with this ideal prior planning. So that it seems finally rather unlikely that 
such a refined behavior of ovipositing females may have developed significantly (at least apart from the specific case of explicitly territorial species). According to this hypothetical and rather unlikely behavior, the density, $h_{i+1}$, of host individuals at generation $i+1$ would simply conform to equation (6) as far as $h_{i+1}$ remains below the carrying capacity $K$ and, beyond, equal this capacity $K$.

A less drastic hypothesis, that yet implies a still rather unlikely pattern of oviposition, leads to the very classic logistic model in discrete time:

$$
h_{i+1}=\left(r . h_{i, \text { non par }}+e_{H}\right) \cdot\left[1-\left(h_{i, \text { non par }}+e_{H}\right) / K\right]
$$

which can provide oscillations of the host and parasite abundances that are not only periodic but also pseudo-periodic or even chaotic.

* now, according to a second, opposite, hypothesis, the pattern of oviposition displayed by hostfemales (or, as well, the pattern of the subsequent distribution of offspring) is assumed being more or less random among the displayed resource. Which implies the absence of any particular effort from host-females to avoid possible intra-specific competition for resource among co-occurring host individuals. This second hypothesis should well deserve more particular attention, as it seems more likely in most host-insects, being understood that, in some case, the field reality may be somewhat an intermediate between these two, opposite, hypothesis.

Assuming thus a (more or less) random distribution of eggs - and, thus, a (more or less) random distribution of offspring among the displayed resource - and then considering the resulting degree of crowding in resource exploitation by host offspring, it comes, in conformity with the Poisson distribution:

$$
h_{i+1}=K \cdot\left[1-\exp \left(-\left(r \cdot h_{i, \text { non par }}+e_{H}\right) / K\right)\right]
$$

That is a more progressive, asymptotic approach of the saturation of resource exploitation quantified by a negative exponential - which is distinctly less brutal than is, for example the linear approach to saturation involved in the logistic model, and still less brutal, a fortiori, than the outcome from the first hypothesis.

Accounting for the expression (2) of $h_{i, \text { non par, }}$ it comes finally:

$$
h_{i+1}=K \cdot\left[1-\exp \left(-\left(r \cdot h_{i} \cdot \exp \left(- \text { a.f. } p_{i}\right)+e_{H}\right) / K\right)\right]
$$

The expression (7) of the density of mated females of parasites, $p_{i+1}$, remains unchanged:

$$
p_{i+1}=v \cdot h_{i \cdot} \cdot\left(1-\exp \left(- \text { a.f. } p_{i}\right)\right)+e_{P}
$$

In contrast with the logistic model evoked above, the present model only generates regular, nonchaotic kinds of oscillations, as a result of the more progressive approach to saturation of resource exploitation by the host. Indeed, this more regular oscillations are in better accordance with many field records.

In addition, note that:

* In the inevitably simplified approach of the model, the interactive dynamics of the host-parasite system is governed by only six main drivers: ' $r$ ' and ' $K$ ' [both relevant to the host]; 'a.f' (whatever the respective values of ' $a$ ' and ' $f$ ') and ' $v$ ' [both relevant to the parasite]; ' $e_{H}$ ' and ' $e_{P}$ ' [both dependent on the environmental biological context]. All or part of these six main drivers being, in turn, variously dependent on the abiotic environmental context (climate, etc...).

* The above equations are given in terms of the areal densities of the parasite $\left(p_{i}\right)$, of the host $\left(h_{i}\right)$ and of the available resource per host $(K=R / c)$. Now, an alternative approach is to consider the respective densities of the host and the parasite relative to the carrying capacity $K$, that is to consider 
$h_{i}^{\prime}=h_{i} / K$ and $p_{i}^{\prime}=p_{i} / K$. This way may arguably be preferred for most practical concerns, as $h_{i}^{\prime}$ more straightforwardly highlights the degree of damage inflict by the host to the feeding resource - damage which, in turn, is often of practical - economic or esthetic - concern. Accordingly, equations (11) and (12) can be rewritten by replacing $h_{i}$ by K. $h_{i}^{\prime} ; p_{i}$ by K. $p_{i}^{\prime} ; e_{H}$ by K.e ${ }_{H}$ and $e_{P}$ by K.e ${ }_{P}$.

It comes accordingly:

and:

$$
h_{i+1}^{\prime}=h_{i+1} / K=1-\exp \left(-\left(r \cdot h_{i}^{\prime} \cdot \exp \left(- \text { a.f.K. } p_{i}^{\prime}\right)+e_{H}^{\prime}\right)\right)
$$

$$
p_{i+1}^{\prime}=p_{i+1} / K=v \cdot h_{i \cdot(}^{\prime}\left(1-\exp \left(- \text { a.f.K. } p_{i}^{\prime}\right)\right)+e_{P}^{\prime}
$$

\section{A.2.3 Dependence of the immigration rates of the parasite and the host on the average densities of parasite and host in the meta-population context}

Up to now, the immigration rates, $e_{H}$ and $e_{p}$, have been considered constant along the time elapsed (i.e. along the successive generations of host and parasite). In particular, no relationship has been considered, that would link the immigration rates of the parasite and the host to the corresponding densities of parasite and host in the more or less distant homologous populations. Yet, it is uncommon that the host and parasite populations are completely isolated from such more or less distant homologous populations. In this "meta-population" context, some exchanges (immigration / emigration) involving both the parasite and the host individuals likely exist between the more or less distant local populations, owing to the unavoidable partial dispersal of individuals away from their native locations. Accordingly, the net immigration rates, $e_{P}$ and $e_{H}$, are expected to represent some definite fraction ' $\varepsilon$ ' of the corresponding local densities $p_{i}$ and $h_{i}$ of the parasite and host (or their respective averages along a cyclic period when cyclic regime actually occurs). This fraction ' $\varepsilon$ ' thus accounts for the relative intensity of the immigration contribution to the dynamics of the interacting host-parasite system under study. Note that, under cyclic regime, the relative contribution $\varepsilon$ of immigration is modulated by the possible partial or even complete synchronization of cycles between the more or less distant homologous host/parasite populations. With $\varepsilon$ decreasing with increasing synchronization and $\varepsilon$ ultimately falling down to zero in the hypothesis of total synchronization.

Let $h^{*}$ and $p^{*}$ be the average values of $p_{i}$ and $h_{i}$ in the meta-population system (i.e. across the neighboring populations); it comes accordingly, at generation i+1:

$$
e_{H}=\varepsilon \cdot h^{*}{ }_{i} \text { and } e_{P}=\varepsilon \cdot p^{*}{ }_{i} \quad \text { or, as well, } \quad e^{\prime}{ }_{H}=\varepsilon \cdot h^{\prime *}{ }_{i} \text { and } e_{P}^{\prime}=\varepsilon \cdot p^{\prime *}{ }_{i}
$$

These expressions having to be introduced in equations (11), (12) or (11bis), (12bis) respectively. Of course, the value of ' $\varepsilon$ ' may possibly differ for the host and for the parasite species.

\section{A.2.4 Implementing the heuristic model}

In spite of its extended parts detailed above, this model remains of course an approximation of a far more complex reality. Yet, as it is, with its additional improvements as compared to the original Nicholson and Bailey model, it offers valuable opportunities of conceptual, speculative investigations and, thereby, may serve as a convenient "heuristic" tool, designed to highlight some influential trends regarding how the interacting dynamics of the host/parasite system actually answers various modifications in the driving parameters ('a', 'f', ' $v$ ', ' $r$ ', ' $K$ ', 'e $e_{P}$ ', ' $e_{H}$ ') of this dynamics.

(c) 2020 Béguinot; This is an Open Access article distributed under the terms of the Creative Commons Attribution License (http://creativecommons.org/licenses/by/4.0), which permits unrestricted use, distribution, and reproduction in any medium, provided the original work is properly cited.

\section{Peer-review history:}

The peer review history for this paper can be accessed here: http://www.sdiarticle4.com/review-history/59302 